\title{
Uso do aplicativo móvel Bone Ninja como ferramenta de avaliação e simulação pré-operatória em pacientes submetidos a osteotomia tibial alta*
}

\section{Use of the Bone Ninja Mobile Application as a Pre- operative Assessment and Simulation Tool in Patients Undergoing High Tibial Osteotomy}

\author{
Rajiv Kaul ${ }^{1}$ (1) Neha Akhoon ${ }^{2}$ (1) \\ 1 Departamento de Ortopedia, Hospital Militar de Kirkee, Pune, India \\ 2 Departamento de Farmacologia, Faculdade de Medicina das Forças \\ Armadas, Pune, India
}

Endereço para Correspondência Rajiv Kaul, MS, Military Hospital Kirkee, Range Hills, Kirkee, Pune, 411020, India (e-mail: drrajivkaul@gmail.com).

Rev Bras Ortop 2022;57(1):89-95.

\section{Resumo \\ Palavras-chave \\ - osteotomia \\ - osteoartrite do joelho \\ - inquéritos e questionários \\ - satisfação do paciente \\ - sistemas de informação em radiologia \\ Objetivo Nosso objetivo foi facilitar a simulação da correção no pré-operatório para permitir a tomada de decisão médico-paciente compartilhada em indivíduos subme- tidos a osteotomia tibial alta (OTA). \\ Método 22 pacientes foram submetidos a osteotomia tibial alta usando dispositivos de fixação internos ou externos para osteoartrite do compartimento medial do joelho. No pré- operatório, a avaliação dos parâmetros de deformidade e a simulação da osteotomia corretiva foram realizadas na presença do paciente, utilizando Bone Ninja. No pós- operatório, o nível de satisfação do paciente com a qualidade da explicação fornecida pelo uso deste software foi avaliado usando o Questionário de Satisfação do Paciente (Patient Satisfaction Questionnaire-short, PSQ-18, na sigla em inglês). Foi realizada uma comparação da correção obtida com recortes de papel e com o software de simulação. \\ Resultados Todos os pacientes ficaram satisfeitos com seu papel no processo de tomada de decisão. Eles mostraram uma boa compreensão e entendimento da cirurgia proposta. Não houve diferença estatisticamente significativamente entre o ângulo tibial proximal medial (ATPM) pré-operatório simulado obtido por recortes de papel e correção assistida por software. O escore médio do PSQ-18 para comunicação foi de 4,24 $(0,88)$, para a qualidade técnica foi de $4,11(0,59)$ e para a satisfação geral foi de $3,11(0,68)$. \\ Conclusão Bone Ninja é uma ferramenta de planejamento de deformidade eficaz, conveniente, fácil de usar e econômica que substitui o método tradicional árduo de traçar no papel e com tesoura.}

Estudo desenvolvido no Departamento de Ortopedia, Hospital Militar Kirkee, Pune, India.

recebido

07 de Maio de 2020

aceito

06 de Julho de 2020

Publicado on-line

Novembro 2, 2020
DOI https://doi.org/

10.1055/s-0040-1716761. ISSN 0102-3616. (c) 2020. Sociedade Brasileira de Ortopedia e Traumatologia. All rights reserved.

This is an open access article published by Thieme under the terms of the Creative Commons Attribution-NonDerivative-NonCommercial-License, permitting copying and reproduction so long as the original work is given appropriate credit. Contents may not be used for commercial purposes, or adapted, remixed, transformed or built upon. (https://creativecommons.org/ licenses/by-nc-nd/4.0/)

Thieme Revinter Publicações Ltda., Rua do Matoso 170, Rio de Janeiro, RJ, CEP 20270-135, Brazil 


\author{
Abstract \\ Keywords \\ - osteotomy \\ - osteoarthritis, knee \\ - surveys and \\ questionnaires \\ - patient satisfaction \\ - radiology \\ information systems
}

Objective Our purpose was to facilitate the simulation of preoperative correction to enable shared doctor-patient decision-making in individuals undergoing high tibial osteotomy (HTO).

Methods A total of 22 patients underwent high tibial osteotomy using internal or external fixation devices for medial compartment osteoarthritis of the knee. Preoperatively, assessment of deformity parameters and simulation of the corrective osteotomy was done in the presence of the patient, using Bone Ninja. Postoperatively, the patient's satisfaction level with the quality of explanation provided by the use of this software was assessed using the Patient Satisfaction Questionnaire-short (PSQ18). A comparison of the correction obtained using paper cuttings and the simulation software was performed.

Results All patients were satisfied with their role in the decision-making process. They showed a good understanding and comprehension of the proposed surgery. There was no statistically significant difference between simulated preoperative Medial Proximal Tibial Angle (MPTA) obtained by paper cuttings and software-assisted correction. The PSQ-18 mean score for communication was $4.24(0.88)$, for technical quality it was 4.11 (0.59) and for general satisfaction it was $3.11(0.68)$.

Conclusion Bone Ninja is an effective, convenient, user-friendly and cost-effective deformity planning tool that supersedes the arduous traditional method of paper tracings and scissors.

\section{Introdução}

A correção da deformidade e a reconstrução de membros estão emergindo rapidamente como uma subespecialidade promissora e futura da ortopedia em todo o mundo. Para corrigir qualquer deformidade, o cirurgião deve primeiro caracterizála e, em seguida, planejar adequadamente uma estratégia corretiva. O papel do planejamento meticuloso pré-operatório e da medição de uma deformidade é fundamental no tratamento de distúrbios do crescimento, mau-alinhamento, uniões mal realizadas e osteoartrite unicompartimental. Isso geralmente é feito com vários sistemas de arquivamento e comunicação de imagens (PACS) e programas de software como TraumaCad (Brainlab, Munique, Alemanha) e PeekMed (PeekMed, Peek Health SA, Braga, Portugal). ${ }^{1}$ Alguns deles são bastante caros e podem não ser facilmente acessíveis a todos. Bone Ninja, um aplicativo móvel, foi desenvolvido para a educação integrada de médicos e pacientes e está disponível na plataforma iPad (iOS). ${ }^{2}$ Esse aplicativo permite que os cirurgiões meçam, planejem e simulem a correção da deformidade sem a necessidade de tesouras, traçados de papel, goniômetros ou programas de software caros.

O objetivo do presente estudo foi facilitar a simulação da correção no pré-operatório para permitir a tomada de decisão compartilhada entre médico e paciente em indivíduos submetidos à Osteotomia Tibial Alta (OTA). Os objetivos foram comparar a correção alcançada usando recortes de papel com a do software de simulação e avaliar o nível de satisfação do paciente e a compreensão do procedimento cirúrgico usando o Questionário de Satisfação do Paciente curto (PSQ-18).

\section{Pacientes e Métodos}

Este foi um estudo prospectivo realizado em um centro ortopédico de atendimento terciário de dezembro de 2016 a junho de 2019 após a liberação do comitê de ética institucional. Um total de 22 pacientes com osteoartrite sintomática do compartimento medial (OACM) do joelho, que foram preparados para a cirurgia corretiva (OTA), foram incluídos no estudo. Foram excluídos aqueles que não foram submetidos a OTA ou que possuíam um banco de dados incompleto.

Todos os pacientes foram submetidos a uma avaliação préoperatória, que incluiu exame clínico de frouxidão ligamentar e mensuração da amplitude de movimento articular e encurtamento dos membros. Foram obtidas radiografias de perna longa e compridas, importadas do departamento de radiologia em um formato de arquivo DICOM, ou, usando a câmera do iPad, uma imagem da radiografia foi clicada e carregada no software Bone Ninja. Além disso, fotografias clínicas de ambos os membros inferiores em pé foram obtidas e armazenadas em um banco de dados computadorizado de pacientes. A sequência do planejamento foi abreviada pelo acrônimo "MAP o $A B C$ ", 2 onde M significa Medir (medindo o desvio do eixo mecânico ou MAD e a discrepância de comprimento do membro), A significa Analisar (os ângulos da articulação/comprimento do osso) e P significa Pegar (o osso a tratar). O primeiro passo foi calibrar o marcador de ampliação usando um círculo de ampliação com a medida de uma polegada $(2,54 \mathrm{~cm})$. 0 cirurgião também pode escolher a ampliação desejada conforme suas necessidades. Em seguida, usando a ferramenta "draw lines"(desenhar linhas), os eixos anatômicos e mecânicos foram desenhados e o desvio do eixo mecânico foi calculado usando a ferramenta "ruler" 


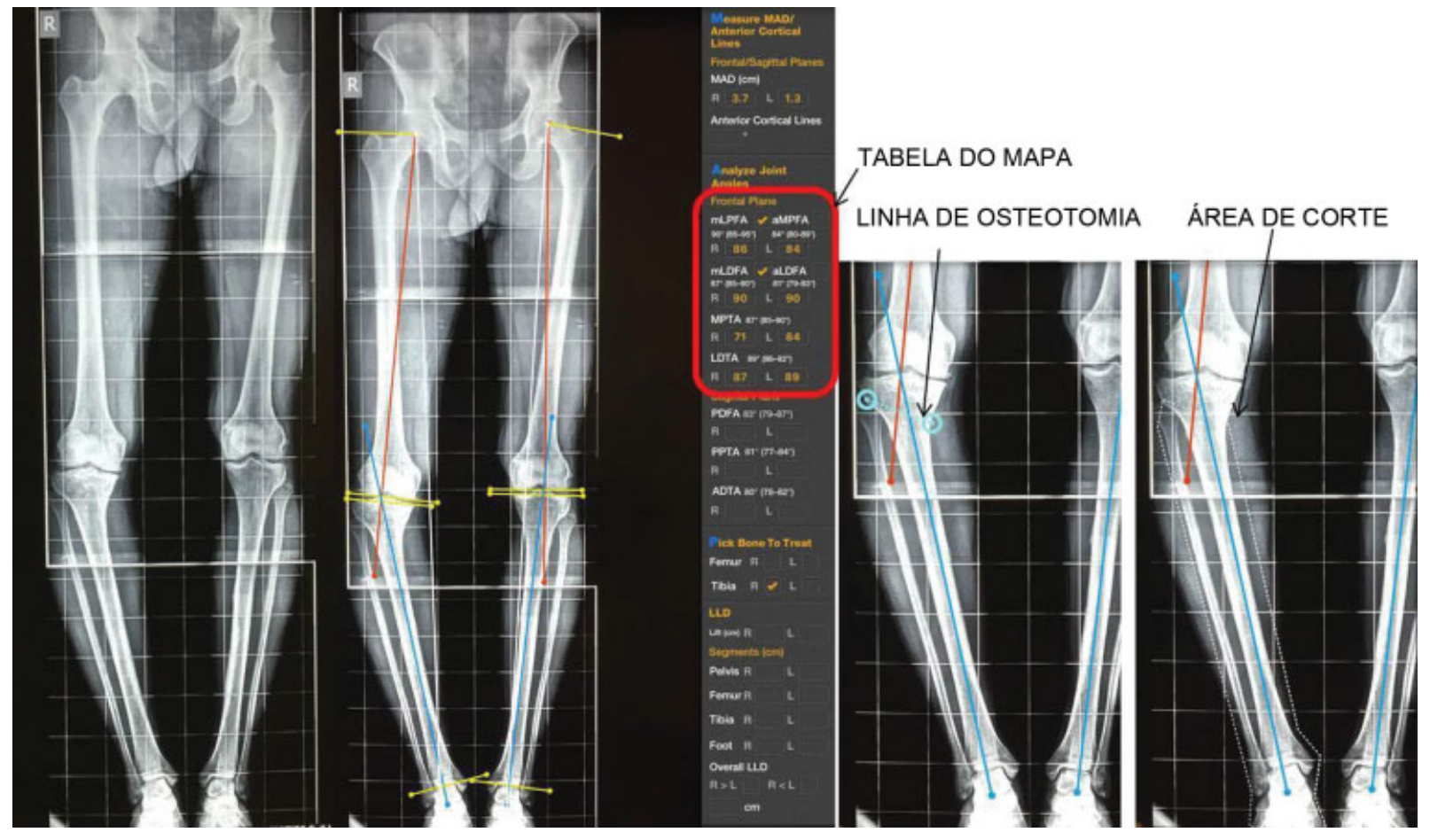

Fig. 1 Marcando linhas e ângulos de orientação da articulação.

(régua). A diferença geral de desalinhamento e comprimento da perna foi registrada. A quantidade de deformidade em varo foi calculada usando a ferramenta "protractor" (transferidor). 0 próximo passo foi marcar as importantes linhas e ângulos de orientação da articulação, como o ângulo mecânico femoral proximal lateral (mLPFA), o ângulo femoral distal lateral (AFDL), $o$ ângulo tibial proximal medial (ATPM) e o ângulo tibial distal lateral (ATDL). ${ }^{3}$ Estes foram registrados na tabela MAP fornecida. (-Fig. 1) Por conveniência, as linhas e ângulos de referência padrão podem ser avaliados a qualquer momento usando a ferramenta "resource"(recurso).

Depois que as medições e análises iniciais foram concluídas, passamos a executar o "ABC", onde A implica determinar o ápice da deformidade, $B$ implica na escolha de um nível de corte ósseo e $C$ representa a escolha de um nível e tipo de correção. $O$ osso afetado era a tíbia e o ápice da deformidade estava localizado no nível da metáfise tibial proximal. Após marcar a linha de osteotomia e selecionar a área de recorte, usamos a ferramenta "thumbtack" (tachinha) para executar um movimento rotacional da área de corte sobre um ponto específico (-Fig. 1). A "thumbtack" foi colocada ao nível da ponta da cabeça fibular para criar uma osteotomia em cunha de abertura medial e junto com ela, o eixo mecânico do segmento distal foi realinhado para coincidir com o eixo mecânico proximal. (-Fig. 2) Realizamos, principalmente, uma super correção, até o ponto Fujisawa. ${ }^{4}$ Uma quantificação da quantidade de correção (base da cunha em milímmetros) foi então realizada usando a 'ruler' (régua). Da mesma forma, uma simulação da osteotomia corretiva foi realizada com as fotografias clínicas, na presença do paciente, o que permitiu ao cirurgião e ao paciente ver a aparência radiográfica e clínica pós-operatória do membro (-Fig. 3).
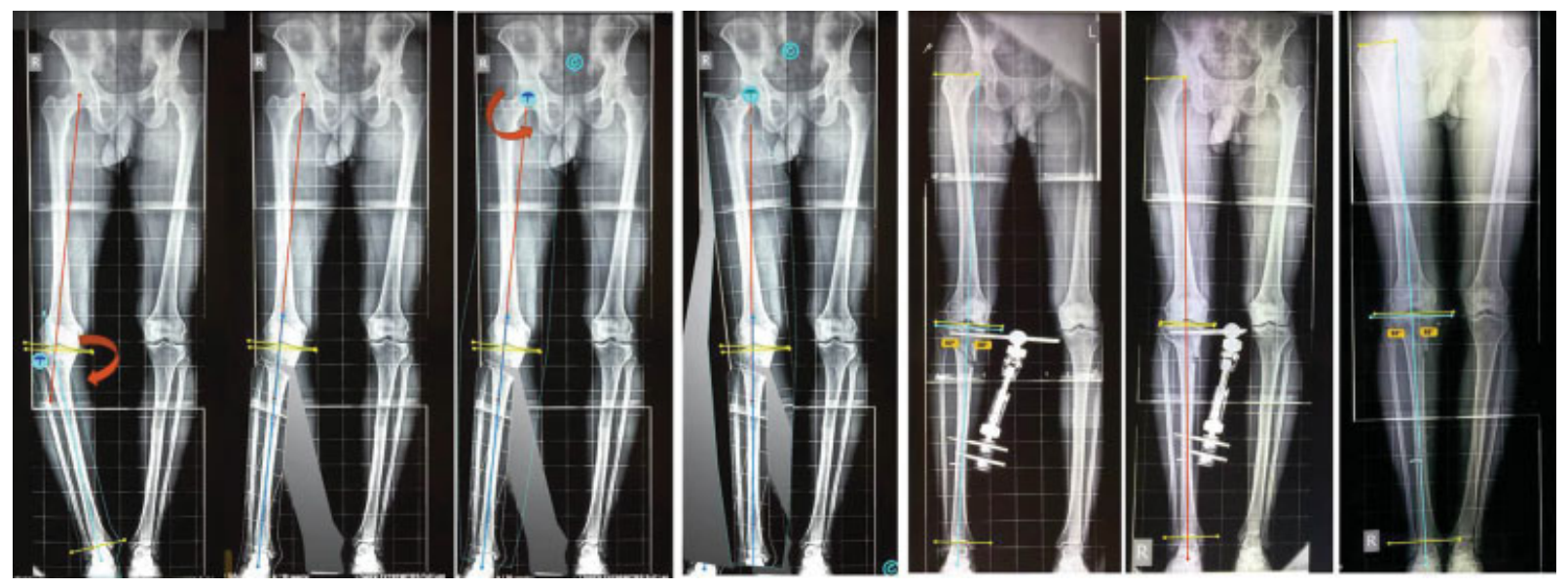

Fig. 2 Simulação de correção usando Bone Ninja. 


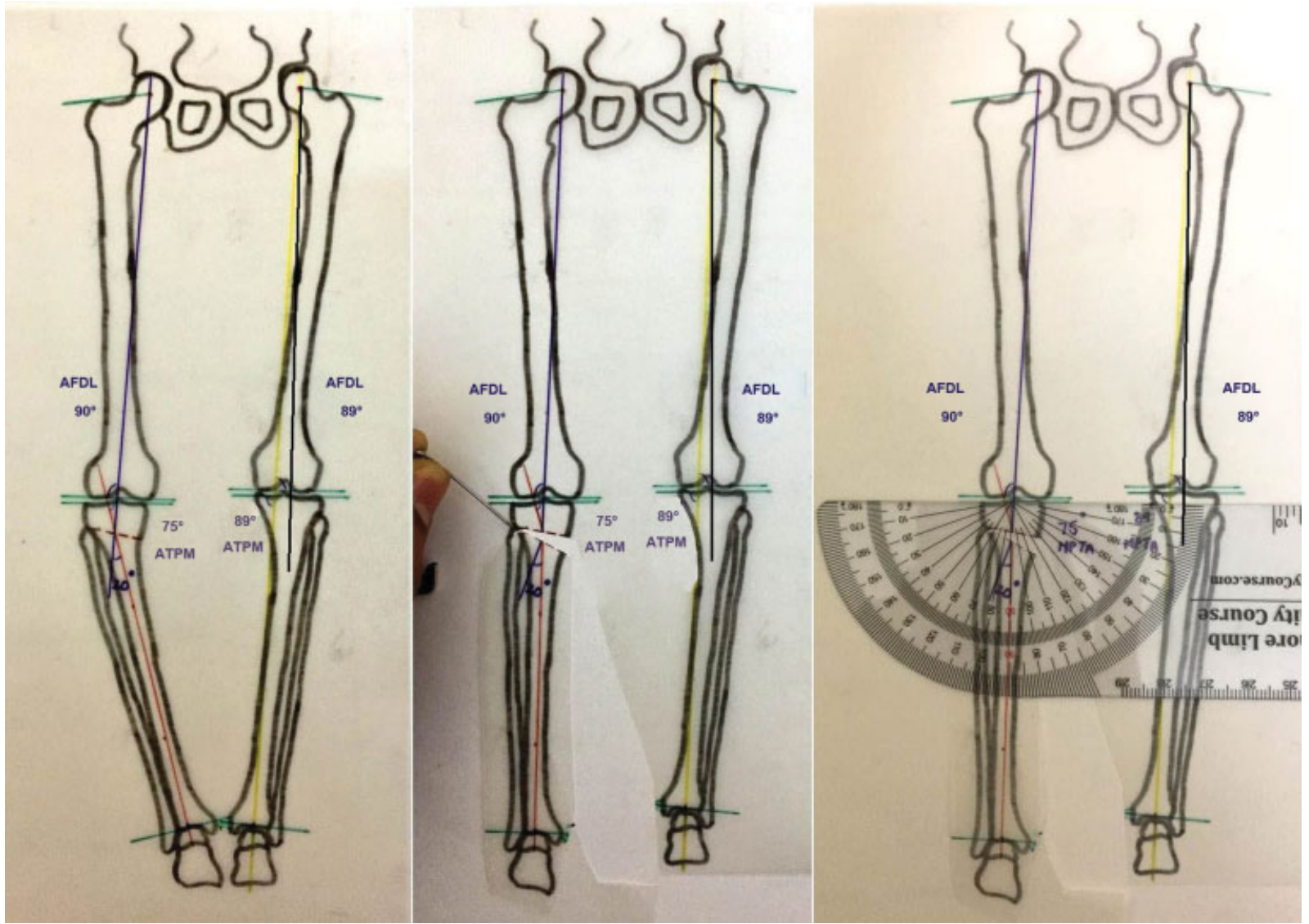

Fig. 3 Simulação de correção usando traçados com papel.

Para fins de comparação, foi desenhado um traçado em papel da radiografia e todas as linhas e ângulos de referência marcados, conforme descrito acima (-Fig. 4). Após traçar a linha da osteotomia, o corte foi feito com tesoura e o fragmento distal girado, colocando um alfinete ou tachinha no nível da cabeça fibular. Após a correção, os pedaços de papel foram colados e os eixos e ângulos foram medidos novamente. 0 ângulo de correção obtido, que é igual à quantidade de correção necessária (assumindo que $1^{\circ}=1 \mathrm{~mm}$ ),foi registrado.

A intervenção cirúrgica realizada foi uma osteotomia tibial alta com cunha de abertura medial (OTA) usando fixador externo poliaxial modular, monolateral (Pitkar Orthotools, Pune, India) ou uma placa de travamento (Tomofix, Synthes, West Chester, PA, EUA). Conforme protocolo, o dispositivo de fixação foi retido por duas vezes o período de distração para facilitar a consolidação da osteotomia. A remoção do fixador foi realizada após a consolidação completa do regenerado, após o qual foi realizada uma análise radiográfica final utilizando o aplicativo. Os ângulos de orientação da articulação foram novamente registrados no banco de dados. A análise estatística para comparação do ATDL pré-operatório obtido após a correção do papel e a obtida pelo software de simulação foram realizadas no Microsoft Excel 2019 (Microsoft Corporation, Redmond, WA, EUA) e o teste utilizado foi o teste $t$ de Student.

O presente trabalho foi aprovado pelo comitê de ética institucional e o consentimento informado foi obtido de todos os participantes individuais incluídos no estudo.
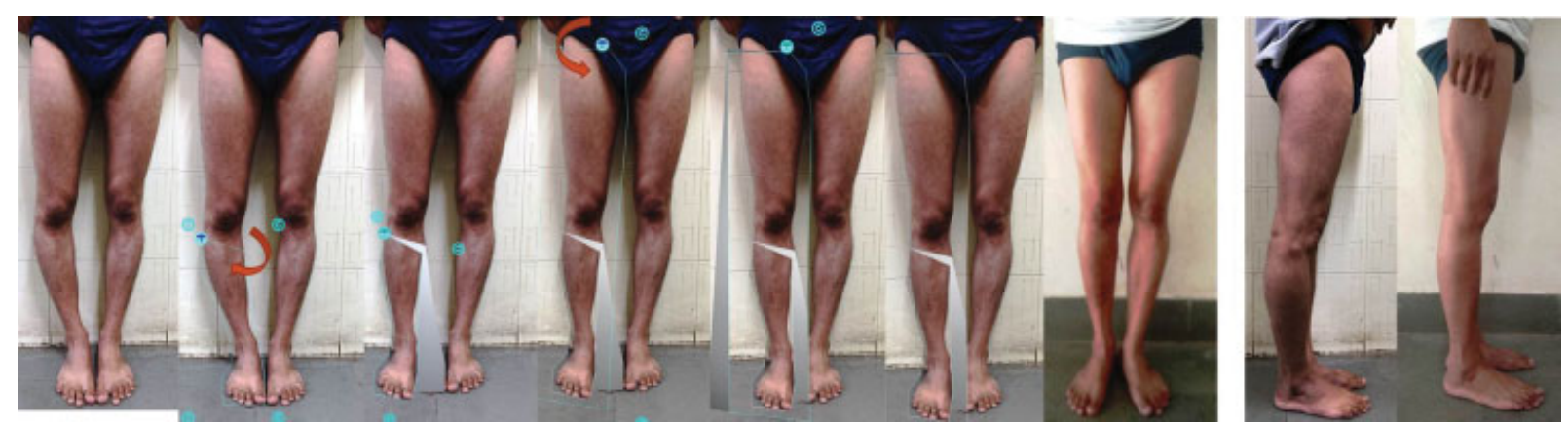

Fig. 4 Simulação de correção na fotografia clínica do paciente. 


\section{Resultados}

Um total de 22 pacientes, incluindo 14 homens e 8 mulheres, submetidos a OTA, foram incluídos no estudo. A idade média dos pacientes foi de $42,45(4,84)$ anos. Todos os pacientes demonstraram bom entendimento e compreensão da cirurgia proposta. Todos ficaram impressionados com a visita inicial e gostaram de ver os resultados simulados pós-correção. Os cirurgiões ficaram satisfeitos com a facilidade de avaliação e registro dos parâmetros radiográficos pré e pós-operatórios usando esta ferramenta. O ATPM médio pré-operatório foi de $74,18^{\circ}(2,26)$, o ATPM médio obtido por corte de papel foi de $87,63^{\circ}(0,78)$, o ATPM médio obtido por simulação de software foi de $87,86^{\circ}(0,94)$ e o ATPM médio após a correção cirúrgica foi de $87,95^{\circ}(0,72)$. O ATPM médio obtido com traçados de papel foi comparado com o ATPM obtido da correção assistida por software e a diferença entre os dois grupos não foi estatisticamente significante $(p=0,29)$ ( - Tabela 2 ).

No momento da alta, todos os pacientes foram solicitados a preencher a versão curta do Questionário de Satisfação do Paciente (PSQ-18), que nos forneceu um feedback sobre seu nível de satisfação ${ }^{5}$ (-Tabela 1 ). A pontuação média para satisfação geral foi de $3,11(0,68)$, a qualidade técnica foi de $4,11(0,59)$, a pontuação média para assuntos interpessoais foi de 3,97 (0,69), a pontuação média de comunicação foi de $4,24(0,88)$, a pontuação média para aspectos financeiros foi de $3,93(0,75)$, a pontuação média para o tempo gasto com o médico foi de $3,45(0,79)$ e a pontuação média para acessibilidade e conveniência foi de $3,84(0,84)$. Mais importante, todos os pacientes estavam muito satisfeitos com seu papel no processo de tomada de decisão.

\section{Discussão}

Tradicionalmente, o planejamento da deformidade é realizado pelo traçado e corte no papel, juntamente com a rotação em torno de um alfinete ou tachinha. Esse processo complicado começou com a preparação de um esboço ou traço dos ossos afetados, seguido de medições usando réguas, transferidores e goniômetros. $^{6,7}$ Essas ferramentas funcionavam bem com raios-X impressos. Na era digital atual, a maioria das radiografias agora são imagens na tela do computador. Lápis e goniômetros não se adaptam bem ao novo meio digital. Isso resultou na evolução de várias ferramentas de Sistema de Arquivamento e Comunicação de Imagens (Picture Archiving and Communication System [PACS, na sigla em inglês]) para planejamento de deformidades. ${ }^{8}$ Com o PACS, há menos chances de uma imagem ser perdida, roubada ou rotulada incorretamente. As ferramentas digitais comumente usadas incluem TraumaCad (Brainlab, Munich, Germany), Adobe Photoshop (Adobe inc., California, USA), and PeekMed (Peek Health SA, Braga, Portugal). ${ }^{11} \mathrm{O}$ objetivo dessa inovação é realizar o planejamento pré-operatório e a simulação dos resultados esperados antes da cirurgia.

O TraumaCad, introduzido em 2005, permite que os cirurgiões avaliem e manipulem imagens digitais enquanto executam o planejamento cirúrgico pré-operatório para osteotomias corretivas e alongamento de membros. ${ }^{9}$ A versão móvel do software foi lançada em 2015. Também possui recursos inte-
Tabela 1 Estatísticas para subescalas PSQ-18 e itens constituintes

\begin{tabular}{|c|c|c|}
\hline Subcategorias PSQ-18 & $\begin{array}{l}\text { Pontuação } \\
\text { média }\end{array}$ & $\mathrm{DP}$ \\
\hline Satisfação Geral & 3,11 & $(0,68)$ \\
\hline $\begin{array}{l}\text { Os cuidados médicos que tenho rece- } \\
\text { bido são praticamente perfeitos }\end{array}$ & 3,27 & 0,63 \\
\hline $\begin{array}{l}\text { Estou insatisfeito com algumas coisas } \\
\text { sobre os cuidados médicos que recebo }\end{array}$ & 2,95 & 0,72 \\
\hline Qualidade técnica & 4,11 & $(0,59)$ \\
\hline $\begin{array}{l}\text { Eu acho que o consultório do meu médico } \\
\text { tem tudo o que é necessário para fornecer } \\
\text { atendimento médico completo }\end{array}$ & 4,04 & 0,57 \\
\hline $\begin{array}{l}\text { Às vezes, os médicos me fazem pensar } \\
\text { se o diagnóstico está correto }\end{array}$ & 4,23 & 0,52 \\
\hline $\begin{array}{l}\text { Quando vou para atendimento médico, } \\
\text { eles têm o cuidado de verificar tudo ao } \\
\text { me tratar e me examinar }\end{array}$ & 4,45 & 0,59 \\
\hline $\begin{array}{l}\text { Tenho algumas dúvidas sobre a capaci- } \\
\text { dade dos médicos que me tratam }\end{array}$ & 4,54 & 0,59 \\
\hline Questão interpessoal & 3,97 & $(0,69)$ \\
\hline $\begin{array}{l}\text { Os médicos agem de maneira muito } \\
\text { impessoal e pragmática em relação a mim }\end{array}$ & 3,95 & 0,65 \\
\hline $\begin{array}{l}\text { Meus médicos me tratam de maneira } \\
\text { amigável e cortês }\end{array}$ & 4 & 0,75 \\
\hline Comunicação & 4,24 & $(0,88)$ \\
\hline $\begin{array}{l}\text { Os médicos são bons em explicar o } \\
\text { motivo dos exames médicos }\end{array}$ & 4,45 & 0,67 \\
\hline $\begin{array}{l}\text { Os médicos às vezes ignoram o que eu } \\
\text { digo a eles }\end{array}$ & 3,22 & 0,61 \\
\hline Aspectos financeiros & 3,93 & $(0,75)$ \\
\hline $\begin{array}{l}\text { Sinto-me confiante de que posso obter } \\
\text { os cuidados médicos de que preciso } \\
\text { sem ter dificuldades financeiras }\end{array}$ & 4,05 & 0,77 \\
\hline $\begin{array}{l}\text { Tenho que pagar por mais assistência } \\
\text { médica do que posso }\end{array}$ & 3,81 & 0,79 \\
\hline Tempo gasto com médico & 3,45 & $(0,79)$ \\
\hline $\begin{array}{l}\text { Quem presta assistência médica às vezes } \\
\text { se apressa demais quando me trata }\end{array}$ & 3,04 & 0,72 \\
\hline $\begin{array}{l}\text { Os médicos geralmente passam muito } \\
\text { tempo comigo }\end{array}$ & 3,86 & 0,63 \\
\hline Acessibilidade e conveniência & 3,84 & $(0,84)$ \\
\hline $\begin{array}{l}\text { Tenho acesso fácil aos médicos espe- } \\
\text { cialistas de que preciso }\end{array}$ & 4,22 & 0,61 \\
\hline $\begin{array}{l}\text { Onde eu recebo atendimento médico, } \\
\text { as pessoas precisam esperar muito } \\
\text { tempo para tratamento de emergência }\end{array}$ & 2,86 & 0,71 \\
\hline $\begin{array}{l}\text { Acho difícil conseguir uma consulta } \\
\text { médica imediatamente }\end{array}$ & 4,13 & 0,56 \\
\hline $\begin{array}{l}\text { Consigo atendimento médico sempre } \\
\text { que preciso }\end{array}$ & 4,13 & 0,63 \\
\hline
\end{tabular}

Abreviação: DP, desvio padrão.

grados, como calculadora de crescimento, módulo de análise de deformidade pediátrica do quadril e um módulo de estrutura espacial especializado de Taylor. Tem sido utilizado para a avaliação da orientação do copo acetabular na prótese de 
Tabela 2 Avaliação comparativa dos resultados

\begin{tabular}{|l|l|l|l|l|}
\hline & Pré-operativo ATPM & ATPM (corte em papel) & ATPM (Bone Ninja) $^{*}$ & Pós-operativo ATPM \\
\hline Média & 74,18 & 87,63 & 87,86 & 87,95 \\
\hline Desvio Padrão & 2,26 & 0,78 & 0,94 & 0,72 \\
\hline $\begin{array}{l}\text { Intervalo de } \\
\text { Confiança (95,0\%) }\end{array}$ & 1,03 & 0,34 & 0,44 & 0,31 \\
\hline Limite Superior & 75,21 & 87,97 & 88,30 & 88,26 \\
\hline Limite Inferior & 73,15 & 87,29 & 87,42 & 87,64 \\
\hline
\end{tabular}

Abreviação: ATPM, ângulo tibial proximal medial.

*o valor de p na comparação entre ATPM (corte de papel) e ATPM (Bone ninja) é calculado usando o teste $t$ de Student como 0,29, o que implica que não há diferença estatisticamente comprovada entre os dois grupos.

recapeamento do quadril por Westacott et al. ${ }^{12}$ e descobriu-se que se correlacionava bem com a tomografia computadorizada (TC). Stefanou et al. ${ }^{13}$ utilizaram o TraumaCad em seu estudo sobre avaliação radiográfica do alongamento dos membros inferiores em pacientes acondroplásticos usando o Quadro de Ilizarov. Segev et al. ${ }^{14}$ relataram boa confiabilidade dessa ferramenta em termos de variabilidade intra e interobservadores e sugeriram seu uso no planejamento de deformidades em ortopedia pediátrica. O planejamento pré-operatório para substituição articular, controle de fraturas e deformidades pediátricas de membros inferiores usando sistemas de software digitalizados, como o TraumaCad, também foi recomendado por Steinberg et al. ${ }^{15}$ Apesar de suas várias vantagens, acreditamos que o alto custo e complexidade do software foram um impedimento para seu uso globalmente.

O aplicativo móvel Bone Ninja é uma ferramenta de ensino desenvolvida por Standard e Herzenberg no Centro Internacional para Alongamento de Membros no Sinai Hospital de Baltimore em 2012. ${ }^{16}$ Foi desenvolvido para ajudar os cirurgiões a aprender a analisar deformidades ósseas longas e simular osteotomias desenhando linhas, medindo ângulos, medindo comprimentos e manipulando fragmentos ósseos. Para iniciantes, estão disponíveis várias lições sobre alinhamento normal dos planos frontal e sagital, deformidades nos planos frontal e sagital, deformidades no plano oblíquo, deformidades de duplo nível, deformidades nos pés e tornozelos, discrepância no comprimento dos membros e alguns capítulos especiais sobre novos métodos de planejamento de deformidades. $\mathrm{O}$ aplicativo também fornece uma solução recomendada para cada lição, para que o cirurgião possa receber feedback, e permite que as lições atuais sejam baixadas da Web. Recursos adicionais incluem a opção de adicionar material virtual, como fixadores monolaterais, placas, parafusos e fixadores de seis eixos à imagem para ajudar a decidir o nível de posicionamento dos elementos transósseos. Hambardzumyan et al. ${ }^{17}$ sugeriram que é uma ferramenta educacional útil, resultando em melhor entendimento e menos conflitos de decisão e expectativas irreais por parte dos pacientes. Whitaker et al. compararam PACS e Bone Ninja para avaliação da discrepância e alinhamento do comprimento dos membros inferiores e não relataram diferença estatística entre as duas modalidades. ${ }^{18}$

No presente estudo, a aplicação Bone Ninja tem sido extremamente benéfica em termos de educação do paciente e preparação do cirurgião. Isso foi avaliado pelas versões mais curta, em inglês e em hindi, do Questionário de Satisfação do Paciente (PSQ-18), que é um questionário de 18 itens que abrange cada um dos 7 aspectos da satisfação com o atendimento médico. ${ }^{19,20}$ Os resultados indicaram que os níveis de satisfação foram maiores para a comunicação, o que implica que os pacientes percebem que o procedimento foi bem explicado, o que pode ter afetado sua tomada de decisão em certa medida. Curvas curtas de aprendizado, recursos fáceis de usar, previsão precisa de correção juntamente com baixos custos fizeram desta aplicação nossa ferramenta preferida de planejamento e avaliação pré-operatória.

Uma possível limitação do presente estudo foi o tamanho da amostra, o que pode justificar a necessidade de estudos adicionais baseados nos níveis de satisfação do paciente.

\section{Conclusão}

A literatura atual apoia o uso de programas de software digital para análise radiográfica de deformidades ortopédicas. Bone Ninja é um software educacional e de planejamento eficaz, conveniente e econômico que substitui o método trabalhoso de traçar em papel e com tesoura. No entanto, até a presente data, existem muito poucos dados publicados para validar o Bone Ninja como uma ferramenta de planejamento para correção de deformidades.

\section{Consentimento Informado}

O consentimento informado foi obtido de todos os participantes individuais incluídos no estudo.

Os autores não estão de forma alguma associados aos fabricantes do software (Sinai Hospital, Baltimore).

\section{Suporte Financeiro}

Não houve apoio financeiro de fontes públicas, comerciais, ou sem fins lucrativos.

\section{Conflito de Interesses}

Os autores não têm conflito de interesses a declarar.

\section{Referências}

1 Strickland NH. PACS (picture archiving and communication systems): filmless radiology. Arch Dis Child 2000;83(01):82-86

2 Standard SC, Herzenberg JE, Conway JD, Lamm BM, Siddiqui NA. The art of limb alignment: International Center for Limb 
Lengthening. Baltimore, Maryland: Rubin Institute for Advanced Orthopedics, Sinai Hospital of Baltimore; 2016

3 Paley D. Principles of Deformity Correction. Berlin: SpringerVerlag; 2014

4 Fujisawa Y, Masuhara K, Shiomi S. The effect of high tibial osteotomy on osteoarthritis of the knee. An arthroscopic study of 54 knee joints. Orthop Clin North Am 1979;10(03):585-608

5 Marshall GN, Hays RD. The patient satisfaction questionnaire short-form (PSQ-18). Santa Monica, CA: RAND Corporation; 1994

6 Kocaoğlu M, Tsuchiya H, Eralp L. Advanced Techniques in Limb Reconstruction Surgery. Berlin: Springer-Verlag; 2014

7 Paccola CAJ. Pre-operative planning and surgical technique of the open wedge supracondylar osteotomy for correction of valgus knee and fixation with a fixed-angle implant. Rev Bras Ortop 2015;45(06):627-635

8 Khakharia S, Bigman D, Fragomen AT, Pavlov H, Rozbruch SR. Comparison of PACS and hard-copy 51-inch radiographs for measuring leg length and deformity. Clin Orthop Relat Res 2011;469(01):244-250

9 TraumaCad orthopaedic pre-operative planning. Available from: https://www.traumacad.com/\#

10 Jamali AA. Digital templating and preoperative deformity analysis with standard imaging software. Clin Orthop Relat Res 2009;467 (10):2695-2704

11 PeekMed, a powerful 3D pre-operative planning software for orthopaedic surgery. Available from: https://www.peekmed.com/\#/

12 Westacott DJ, McArthur J, King RJ, Foguet P. Assessment of cup orientation in hip resurfacing: a comparison of TraumaCad and computed tomography. J Orthop Surg Res 2013;8(01):8
13 Stefanou MA, Pasparakis D, Mastrokalos D, Tzoumaka-Bakoula C, Papandreou N, Papagelopoulos PJ. Radiographic assessment of lower limb lengthening in achondroplastic patients, using the ilizarov frame: a 5-19 year follow up study. Int J Orthod 2014;1 (04):140-145

14 Segev E, Hemo Y, Wientroub S, et al. Intra- and interobserver reliability analysis of digital radiographic measurements for pediatric orthopedic parameters using a novel PACS integrated computer software program. J Child Orthop 2010;4(04):331-341

15 Steinberg EL, Segev E, Drexler M, Ben-Tov T, Nimrod S. Preoperative planning of orthopedic procedures using digitalized software systems. Isr Med Assoc J 2016;18(06):354-358

16 Bone Ninja application for iPad. Available from: http://www. limblength.org/about-us/physician-education/bone-ninja-appfor-ipad

17 Hambardzumyan V, Herzenberg JE. Bone Ninja app as a body image simulation tool for shared decision-making. J Limb Lengthening Reconstr 2019;5(02):105

18 Whitaker AT, Gesheff MG, Jauregui JJ, Herzenberg JE. Comparison of PACS and Bone Ninja mobile application for assessment of lower extremity limb length discrepancy and alignment. J Child Orthop 2016;10(05):439-443

19 Thayaparan AJ, Mahdi E. The Patient Satisfaction Questionnaire Short Form (PSQ-18) as an adaptable, reliable, and validated tool for use in various settings. Med Educ Online 2013;18:21747

20 Shrivastava M, Shah N, Dixit A, Pandey M. Hindi Translation and Cross-Cultural Reliability and Validity of Patient Satisfaction Questionnaire Short Form (PSQ-18). World Journal of PsychoSocial Oncology 2017;4(01) 